\title{
Geometric properties of the meromorphic functions class through special functions associated with a linear operator
}

\author{
F. Ghanim ${ }^{1 *}$, Hiba F. Al-Janaby² and Omar Bazighifan ${ }^{3}$ (B)
}

\section{"Correspondence:}

fgahmed@sharjah.ac.ae

'Department of Mathematics,

College of Sciences, University of

Sharjah, Sharjah, United Arab

Emirates

Full list of author information is

available at the end of the article

\begin{abstract}
According to the theory of regular geometric functions, the relevance of geometry to analysis is a critical feature. One of the significant tools to study operators is to utilize the convolution product. The dynamic techniques of convolution have attracted numerous complex analyses in current research. In this effort, an attempt is made by utilizing the said techniques to study a new linear complex operator connecting an incomplete beta function and a Hurwitz-Lerch zeta function of certain meromorphic functions. Furthermore, we employ a method based on the first-order differential subordination to derive new and better differential complex inequalities, namely differential subordinations.
\end{abstract}

MSC: 30D30; $11 \mathrm{M} 35 ; 30 \mathrm{C} 80$

Keywords: Meromorphic function; Convolution product; Hurwitz-Lerch zeta function; Differential first-order subordination

\section{Introduction}

During the nineteenth century, the theory of regular geometric functions, which is an eminent field of complex analysis, was first originated by Riemann in 1850. It was amended in 1907 by Koebe who introduced the concept of univalent functions in his monograph. This theory deals with various sorts of normalized univalent regular functions in the open unit disk, such as $p$-valent, meromorphic, meromorphic $p$-valent, harmonic, and fractional regular functions. Later, due to its great significance, it was developed and formulated by famed mathematicians such as Lindelŏf [1], who put forward the term of subordination in the complex plane between regular functions, which is the generalization formula for inequality on the real line. The idea of subordination has been utilized to investigate numerous classes of functions studied in the theory of regular geometric functions. Over the years, Miller and Mocanu [2, 3] in 2000 and 2003, respectively, introduced and developed the differential subordination theory. In this context, operator theory is closely related to the theory of regular geometric functions, special function theory, the latter being one of the most studied areas of mathematics due to its numerous applications. In other words, the study of linear and nonlinear operators is a crucial and delightful tool for attempting

(c) The Author(s) 2022. This article is licensed under a Creative Commons Attribution 4.0 International License, which permits use sharing, adaptation, distribution and reproduction in any medium or format, as long as you give appropriate credit to the original author(s) and the source, provide a link to the Creative Commons licence, and indicate if changes were made. The images or other third party material in this article are included in the article's Creative Commons licence, unless indicated otherwise in a credit line to the material. If material is not included in the article's Creative Commons licence and your intended use is not permitted by statutory regulation or exceeds the permitted use, you will need to obtain permission directly from the copyright holder. To view a copy of this licence, visit http://creativecommons.org/licenses/by/4.0/. 
to generalize and define a lot of new geometric classes of regular functions associated with special functions. Another tool, such as convolution product (Hadamard product), constructed by Hadamard [1] in 1899, is important to write many differential and integral operators in terms of convolution of certain regular functions. These formalities bring ease in further mathematical explanation. The first integral operator was studied by Alexander [4] in 1915. This operator was termed Alexander integral operator. Afterward, in 1965, Libera [5] presented an integral operator and discussed specified properties of starlike functions under this operator. In 1975, Ruscheweyh [6] studied and presented the differential operator based on the convolution product technique for a class of regular functions. In 1975 and in 1990, the nonlinear integral operator was investigated and introduced by Pfaltzgraff [7] and Pascu and Pescar [8], respectively. This operator is called an integral of the second type. In 1983, another version of the differential operator (linear operator) was considered by Sălăgean [9], namely Salagean differential operator. In 1984, Carlson and Shaffer [10] proposed a convolution linear operator in terms of a certain class of special functions including incomplete beta functions. Corresponding formulations were discussed in 1989 by Srivastava and Owa [11], and they imposed the nonlinear types of fractional operators (derivative and integral) in the complex plane, called fractional calculus operators in the sense of Srivastava and Owa operators. Since then, the interest in utilizing classes of special functions has vastly increased till the present day. One of the basically higher transcendental functions is the Hurwitz-Lerch zeta (HL-Z) function. The author Srivastava has contributed significantly to the study of numerous features, extensions, and generalizations of the HL-Z unction. For their main contributions, in 2007, Srivastava and Attiya [12] first utilized and studied the HL-Z function to introduce an integral operator on several subclasses of the class of normalized regular functions. This operator is called Srivastava-Attiya operator. In 2011, Srivastava et al. [13] introduced a systematic investigation of various integrals and computational representations for several classes of generalized HL-Z functions. Furthermore, they studied their relationship with the $\mathrm{H}$-function, which derived the Mellin-Barnes type integral representations for nearly all of the generalized and specialized HL-Z functions. In addition, fractional derivatives related to the generalized HL-Z functions were also examined. The relationship between the generalized HL-Z function and the H-function has been given its corrected version. Unification and extension of the HL-Z function and two of its special cases connected with the MittagLeffler type functions were discussed. In 2019, Srivastava [14, 15] provided an overview of several recent evolutions involving the Riemann zeta (RZ) function, the Hurwitz zeta (HZ) function and its generalized function, and the HL-Z function. In the same year, Srivastava [16] systematically investigated several classes of the HL-Z function that include the so-called $\lambda$-generalized HL-Z functions. In 2020, Srivastava [17] exposed classes of such widely discussed Srivastava-Attiya operators along with their generalized and extended versions. In 2021, Srivastava et al. [18] utilized the q-Srivastava-Attiya operator to present a new class of normalized regular and bi-univalent functions correlating with the Bazilevič functions and the $\lambda$-pseudo-starlike functions along with the Horadam polynomials. They utilized the basic or quantum (or q-) extension of the class of HL-Z functions which possess features with local or nonlocal symmetries to study the implementations of such said functions that are motivated by the symmetric nature of quantum calculus itself. Actually, the connection between the theory of regular geometric functions and special 
function theory is very interesting and, therefore, other complex analysts who have made significant contributions to the study of the related problems are such as [19-31], and [32].

Motivated by the above-mentioned previous works, in this study we impose a new linear complex operator associating incomplete beta functions with the HL-Z function of certain meromorphic functions by employing the convolution techniques. Moreover, certain outcomes concerning the implementation of first-order differential subordination for a new class are examined, including the said operator.

Since then, numerous techniques have evolved to study diverse classes of HL-Z and related functions. Among things, some classes of special functions were formulated by utilizing the theory of gamma functions and beta functions. The significant contribution of this work is the enrichment of some areas of mathematics like operator theory closely related to special function theory, and the convolution linear operator in terms of the HL-Z function is introduced. The regular geometric functions of meromorphic functions, which are their generalized well-known subclasses, are defined. Some inequalities including linear complex operators are imposed by employing differential subordination. In this study, we deal with the generalized meromorphic HL-Z function structure and convolution product to investigate a certain class of operators involved in geometric function theory.

In this mathematical study, the class of regular meromorphic functions $f(z)$ is a crucial gadget in developing this discipline and in almost all fields of mathematics. This class is denoted by $\Sigma$ and proposed as follows:

$$
f(z)=\frac{1}{z}+\sum_{n=1}^{\infty} a_{n} z^{n}
$$

which are univalent in a complex punctured disk $\mathfrak{D}^{*}=\{z: 0<|z|<1\}$. The complex unit disk is symbolized by $\mathfrak{D}=\{z:|z|<1\}$. The functions $f_{\ell}(z) \in \Sigma$, for $\ell=1$, 2 , are defined as

$$
f_{\ell}(z)=\frac{1}{z}+\sum_{n=1}^{\infty} a_{n, \ell} z^{n} .
$$

Further, the convolution products of $f_{1}(z)$ and $f_{2}(z)$ are formulated as

$$
\left(f_{1} * f_{2}\right)=\frac{1}{z}+\sum_{n=1}^{\infty} a_{n, 1} a_{n, 2} z^{n}
$$

For $\varrho \in \mathcal{C} \backslash\{0\} ; \tau \neq 0,-1,-2, \ldots$, we impose the following special function $E(\varrho, \tau ; z)$ :

$$
E(\varrho, \tau ; z)=\frac{1}{z}+\sum_{n=0}^{\infty} \frac{(\varrho)_{n+1}}{(\tau)_{n+1}} z^{n}
$$

where $(\chi)_{n}=\chi(\chi+1)_{n+1}$ is the Appell symbol or, namely, the Pochhammer symbol. Notice that

$$
E(\varrho, \tau ; z)=\frac{1}{z} 2 \mathfrak{F}_{1}(1, \varrho, \tau ; z),
$$


where

$$
{ }_{2} \mathfrak{F}_{1}(\mu, \varrho, \tau ; z)=\sum_{n=0}^{\infty} \frac{(\mu)_{n}(\varrho)_{n}}{(\tau)_{n}} \frac{z^{n}}{n !}
$$

is the famed hypergeometric function. Moreover, we recall a more general special function, namely Hurwitz-Lerch zeta (HL-Z) function, which was introduced in [12, 33]:

$$
\mathfrak{P}(z, \varsigma, \sigma)=\frac{1}{\sigma^{\varsigma}}+\sum_{n=1}^{\infty} \frac{z^{n}}{(n+\sigma)^{\varsigma}}
$$

$\left(\sigma \in \mathcal{C} \backslash\{0,-1, \ldots\} ; \varsigma \in \mathcal{C}\right.$ when $z \in \mathfrak{D}=\mathfrak{D}^{*} \cup\{0\} ; \mathfrak{R}(\varsigma)>1$ when $\left.z \in \partial \mathfrak{D}\right)$.

Considerable special cases for $\mathfrak{P}(z, \varsigma, \sigma)$ involve the Riemann zeta function $\psi(\varsigma)=$ $\mathfrak{P}(1, \varsigma, 1)$, the Hurwitz zeta function $\psi(\varsigma, \sigma)=\mathfrak{P}(1, \varsigma, \sigma)$, the Lerch zeta function $l_{\varsigma}(\psi)=$ $\mathfrak{P}\left(\exp ^{2 \pi i \zeta}, \varsigma, 1\right),(\zeta \in \mathbb{R}, \mathfrak{R}(\varsigma)>1)$, the polylogarithm $L_{\varsigma}^{i}(z)=z \mathfrak{P}(z, \varsigma, \sigma)$, and others. For recent outcomes on $\mathfrak{P}(z, \varsigma, \sigma)$, see [34, 35], and [36]. By utilizing equation (1.4), we introduce

$$
\begin{aligned}
G_{\zeta, \sigma}(z) & =(1+\sigma)^{\varsigma}\left[\mathfrak{P}(z, \varsigma, \sigma)-\sigma^{\varsigma}+\frac{1}{z(1+\sigma)^{\varsigma}}\right] \\
& =\frac{1}{z}+\sum_{n=1}^{\infty}\left(\frac{1+\sigma}{n+\sigma}\right)^{\varsigma} z^{n}, \quad\left(z \in \mathfrak{D}^{*}\right) .
\end{aligned}
$$

Corresponding to $E(\varrho, \tau ; z), G_{5, \sigma}(z)$ is given by (1.4) and (1.6) consistently, and utilizing the convolution product for $f \in \Sigma$, we provide the following convolution (linear) operator $\mathcal{L}_{\zeta, \sigma}(\varrho, \tau)$ on $\Sigma$ :

$$
\begin{aligned}
\mathcal{L}_{\sigma}^{\varsigma}(\varrho, \tau) f(z) & =E(\varrho, \tau ; z) * G_{5, \sigma}(z) * f(z) \\
& =\frac{1}{z}+\sum_{n=1}^{\infty} \frac{(\varrho)_{n+1}}{(\tau)_{n+1}}\left(\frac{1+\sigma}{n+\sigma}\right)^{\varsigma} a_{n} z^{n}, \quad\left(z \in \mathfrak{D}^{*}\right) .
\end{aligned}
$$

Following this, we introduce a certain new class for meromorphic functions containing the convolution linear operator (1.7).

Definition 1.1 For $\varpi, \eta(-1 \leq \eta<\varpi \leq 1)$ and $0 \leq \kappa<1$, the function $f \in \Sigma$ is a subset of the class $\Lambda_{\sigma}^{5}(\varrho, \tau ; \kappa ; \varpi, \eta)$ if it achieves the subsequent stipulation:

$$
\frac{1}{1-\kappa}\left(\frac{-z\left(\mathcal{L}_{\sigma}^{\varsigma}(\varrho, \tau) f(z)\right)^{\prime}}{z\left(\mathcal{L}_{\sigma}^{\varsigma}(\varrho, \tau) f(z)\right)}-\kappa\right) \prec \frac{1+\varpi z}{1+\eta z} \quad\left(z \in \mathfrak{D}^{*}\right)
$$

\section{Preliminary outcomes}

This section reviews the lemmas which will be employed to acquire our main consequence.

Lemma 2.1 Let $f \in \Sigma, \varrho, \tau \in \mathcal{C}$ such that $\varrho, \tau \notin \mathcal{Z}_{0}^{-}, \kappa, \varsigma \in \mathcal{N}, \vartheta>0, \sigma \in \mathbb{R}$. Then the following identities hold: 
1.

$$
z\left(\mathcal{L}_{\sigma}^{\varsigma}(\varrho+1, \tau) f(z)\right)^{\prime}=\varrho\left(\mathcal{L}_{\sigma}^{\varsigma}(\varrho, \tau) f(z)\right)-(\varrho+1) \mathcal{L}_{\sigma}^{\varsigma}(\varrho+1, \tau) f(z)
$$

2.

$$
z\left(\mathcal{L}_{\sigma}^{\varsigma}(\varrho, \tau) f(z)\right)^{\prime}=\tau\left(\mathcal{L}_{\sigma}^{\varsigma}(\varrho, \tau+1) f(z)\right)-(\tau+1) \mathcal{L}_{\sigma}^{\varsigma}(\varrho, \tau) f(z)
$$

3.

$$
z\left(\mathcal{L}_{\sigma}^{\zeta, \kappa}(\varrho, \tau) f(z)\right)^{\prime}=\frac{1}{\vartheta}(1-\vartheta) \mathcal{L}_{\sigma}^{\zeta, \kappa}(\varrho, \tau) f(z)-\frac{1}{\vartheta} \mathcal{L}_{\sigma}^{\zeta+1, \kappa}(\varrho, \tau) f(z)
$$

4.

$$
z\left(\mathcal{L}_{\sigma}^{\varsigma}(\varrho+1, \tau) f(z)\right)^{\prime}=\varrho \mathcal{L}_{\sigma}^{\varsigma}(\varrho, \tau) f(z)-(\varrho+1) \mathcal{L}_{\sigma}^{\varsigma}(\varrho+1, \tau) f(z),
$$

5.

$$
z\left(\mathcal{L}_{\sigma}^{\varsigma}(\varrho, \tau) f(z)\right)^{\prime}=(\sigma+1) \mathcal{L}_{\sigma+1}^{\varsigma}(\varrho, \tau) f(z)-(\sigma+2) \mathcal{L}_{\sigma}^{\varsigma}(\varrho, \tau) f(z)
$$

6.

$$
z\left(\mathcal{L}_{\sigma}^{\zeta}(\varrho, \tau) f(z)\right)^{\prime}=\tau \mathcal{L}_{\sigma}^{\zeta}(\varrho, \tau+1) f(z)-(\tau+1) \mathcal{L}_{\sigma}^{\varsigma}(\varrho, \tau) f(z) .
$$

Proof By using series expansions of separate functions, these identities can be attained.

Lemma 2.2 ([37]) Let $\omega$ be a univalent function in $\mathfrak{D}$, and let $\varphi$ and $\rho$ be regular in a domain D including $\omega(\mathfrak{D})$ with $\rho(\mathfrak{a}) \neq 0$ when $\mathfrak{a} \in \omega(\mathfrak{D})$. Set $\mathcal{H}(z)=z \omega^{\prime}(z) \rho(\omega(z))$ and $\mathfrak{q}(z)=$ $\varphi(\omega(z))+\mathcal{H}(z)$. Consider that

(i) $\mathcal{H}$ is starlike univalent in $\mathfrak{D}$,

(ii) For $z \in \mathfrak{D}, \Re\left(\frac{z \mathfrak{q}^{\prime}(z)}{\mathcal{H}(z)}\right)>0$. If $\mathfrak{t}$ is regular with $\mathfrak{t}(0)=\omega(0), \mathfrak{t}(\mathfrak{D}) \subseteq D$ and

$$
\varrho(\mathfrak{t}(z))+z \mathfrak{t}^{\prime}(z) \rho(\mathfrak{t}(z)) \prec \varrho(\omega(z))+z \omega^{\prime}(z) \rho(\omega(z))
$$

then

$$
\mathfrak{t}(z) \prec \omega(z) \quad(z \in \mathfrak{D})
$$

and $\omega$ is the best dominant.

Lemma 2.3 ([38]) Let $\omega$ be a convex univalent in $\mathfrak{D}$, and let $\tau \in \mathcal{C}, \pi \in \mathcal{C}^{*}=\mathcal{C} \backslash\{0\}$ with

$$
\Re\left\{1+\frac{z \omega^{\prime \prime}(z)}{\omega^{\prime}(z)}\right\}>\max \left\{0,-\Re\left(\frac{\tau}{\pi}\right)\right\} .
$$

If $\mathfrak{t}$ is regular in $\mathfrak{D}$ with $\mathfrak{t}(0)=h(0)$ and

$$
\tau \mathfrak{t}(z)+\pi z \mathfrak{t}^{\prime}(z) \prec \tau \omega(z)+\pi z \omega^{\prime}(z)
$$


then

$$
\mathfrak{t}(z) \prec \omega(z) \quad(z \in \mathfrak{D})
$$

and $\omega$ is the best dominant.

\section{Geometric features}

Theorem 3.1 Let $\pi \in \mathcal{C}^{*}$. If $\in \Sigma$ and $\mathcal{J}$ is convex univalent in $\mathfrak{D}$ with $\mathcal{J}(0)=1$. Iff and $\mathcal{J}$ attain any one of the following pairs of stipulation:

1.

$$
\begin{aligned}
& \Re\left\{1+\frac{z \mathcal{J}^{\prime \prime}(z)}{\mathcal{J}^{\prime}(z)}\right\}>\max \left\{0, \frac{1}{\xi} \Re\left(\frac{1}{\pi}\right)\right\}, \\
& \pi\left(z \mathcal{L}_{\sigma}^{\mathcal{S}+1}(\varrho, \tau) f(z)\right)+(1-\pi)\left(z \mathcal{L}_{\sigma}^{\varsigma}(\varrho, \tau) f(z)\right) \prec \mathcal{J}(z)-\pi \vartheta z \mathcal{J}^{\prime}(z), \quad z \in \mathfrak{D},
\end{aligned}
$$

2.

$$
\begin{aligned}
& \Re\left\{1+\frac{z \mathcal{J}^{\prime \prime}(z)}{\mathcal{J}^{\prime}(z)}\right\}>\max \left\{0, \Re\left(\frac{\varrho-1}{\pi}\right)\right\}, \\
& \pi\left(z \mathcal{L}_{\sigma}^{\zeta}(\varrho-1, \tau) f(z)\right)+(1-\pi)\left(z \mathcal{L}_{\sigma}^{\varsigma}(\varrho, \tau) f(z)\right) \prec \mathcal{J}(z)-\frac{\pi}{\varrho-1} z \mathcal{J}^{\prime}(z), \\
& \quad z \in \mathfrak{D},
\end{aligned}
$$

3.

$$
\begin{aligned}
& \Re\left\{1+\frac{z \mathcal{J}^{\prime \prime}(z)}{\mathcal{J}^{\prime}(z)}\right\}>\max \left\{0,-(\pi+1) \Re\left(\frac{1}{\pi}\right)\right\}, \\
& \lambda\left(z \mathcal{L}_{\sigma+1}^{\varsigma}(\varrho, \tau) f(z)\right)+(1-\pi)\left(z \mathcal{L}_{\sigma}^{\varsigma}(\varrho, \tau) f(z)\right) \prec \mathcal{J}(z)-\frac{\pi}{\sigma+1} z \mathcal{J}^{\prime}(z), \\
& \quad z \in \mathfrak{D},
\end{aligned}
$$

4.

$$
\begin{aligned}
& \Re\left\{1+\frac{z \mathcal{J}^{\prime \prime}(z)}{\mathcal{J}^{\prime}(z)}\right\}>\max \left\{0, \Re\left(\frac{\tau}{\pi}\right)\right\}, \\
& \pi\left(z \mathcal{L}_{\sigma}^{\varsigma}(\varrho, \tau+1) f(z)\right)+(1-\pi)\left(z \mathcal{L}_{\sigma}^{\varsigma}(\varrho, \tau) f(z)\right) \prec \mathcal{J}(z)-\frac{\pi}{\tau} z \mathcal{J}^{\prime}(z), \quad z \in \mathfrak{D} .
\end{aligned}
$$

Then

$$
z \mathcal{L}_{\sigma}^{\zeta}(\varrho, \tau) f(z) \prec \mathcal{J}(z)
$$

and $\mathcal{J}$ is the best dominant of (3.9).

Proof Differentiating the following function:

$$
\mathcal{H}(z)=z \mathcal{L}_{\sigma}^{\varsigma}(\varrho, \tau) f(z), \quad z \in \mathfrak{D},
$$


by using identities (2.1)-(2.6), we consistently gain the following:

$$
\begin{aligned}
& z \mathcal{L}_{\sigma}^{\varsigma+1}(\varrho, \tau) f(z)=\mathcal{H}(z)-\vartheta z \mathcal{H}^{\prime}(z), \\
& z \mathcal{L}_{\sigma}^{\varsigma}(\varrho-1, \tau) f(z)=\mathcal{H}(z)+\frac{1}{\varrho-1} z \mathcal{H}^{\prime}(z), \\
& z \mathcal{L}_{\sigma+1}^{\varsigma}(\varrho, \tau) f(z)=\mathcal{H}(z)+\frac{z \mathcal{H}^{\prime}(z)}{1+\sigma},
\end{aligned}
$$

and

$$
z \mathcal{L}_{\sigma}^{\varsigma}(\varrho, \tau+1) f(z)=\mathcal{H}(z)+\frac{1}{\tau} z \mathcal{H}^{\prime}(z) .
$$

Now, subordination stipulations (3.2), (3.4), (3.6), and (3.8) are consistently equivalent to

$$
\begin{aligned}
& \mathcal{H}(z)-\pi \vartheta z \mathcal{H}^{\prime}(z) \prec \mathcal{J}(z)-\pi \vartheta z \mathcal{J}^{\prime}(z), \\
& \mathcal{H}(z)+\frac{\pi}{\varrho-1} z \mathcal{H}^{\prime}(z) \prec \mathcal{J}(z)+\frac{\pi}{\varrho-1} z \mathcal{J}^{\prime}(z), \\
& \mathcal{H}(z)+\frac{\pi}{\sigma+1} z \mathcal{H}^{\prime}(z) \prec \mathcal{J}(z)+\frac{\pi}{\sigma+1} z \mathcal{J}^{\prime}(z),
\end{aligned}
$$

and

$$
\mathcal{H}(z)+\frac{\pi}{\tau} z \mathcal{H}^{\prime}(z) \prec \mathcal{J}(z)++\frac{\pi}{\tau} z \mathcal{J}^{\prime}(z)
$$

Successively, by employing Lemma 2.3 to each of the subordination stipulations (3.13)(3.17) with suitable choices of $\tau$ and $\pi$, we gain assertion (3.9) of Theorem 3.1.

Theorem 3.2 Let $\pi \in \mathcal{C}^{*},-1 \leq \eta<\varpi \leq 1$, and $f \in \Sigma$. If any one of the following pairs of stipulations is attained:

1 .

$$
\begin{aligned}
& \frac{|\eta|-1}{|\eta|+1}<\frac{-1}{\vartheta} \Re\left(\frac{1}{\pi}\right) \\
& \pi\left(z \mathcal{L}_{\sigma}^{\varsigma+1}(\varrho, \tau) f(z)\right)+(1-\lambda)\left(z \mathcal{L}_{\sigma}^{\varsigma}(\varrho, \tau) f(z)\right) \prec \frac{1+\varpi z}{1+\eta z}+\pi \vartheta \frac{(\varpi-\eta) z}{(1+\eta z)^{2}}
\end{aligned}
$$

2.

$$
\begin{aligned}
& \frac{|\eta|-1}{|\eta|+1}<\mathfrak{R}\left(\frac{\varrho-1}{\pi}\right) \\
& \pi\left(z \mathcal{L}_{\sigma}^{\varsigma}(\varrho-1, \tau) f(z)\right)+(1-\pi)\left(z \mathcal{L}_{\sigma}^{\varsigma}(\varrho, \tau) f(z)\right) \\
& \quad \prec \frac{1+\varpi z}{1+\eta z}+\frac{(\varpi-\eta) \pi z}{(\varrho-1)(1+\eta z)^{2}},
\end{aligned}
$$

3.

$$
\frac{|\eta|-1}{|\eta|+1}<(\sigma+1) \Re\left(\frac{1}{\pi}\right),
$$




$$
\pi\left(z \mathcal{L}_{\sigma+1}^{\varsigma}(\varrho, \tau) f(z)\right)+(1-\pi)\left(z \mathcal{L}_{\sigma}^{\varsigma}(\varrho, \tau) f(z)\right) \prec \frac{1+\varpi z}{1+\eta z}+\frac{(\varpi-\eta) \pi z}{(\sigma+1)(1+\eta z)^{2}}
$$

4.

$$
\begin{aligned}
& \frac{|\eta|-1}{|\eta|+1}<\Re\left(\frac{\tau}{\pi}\right), \\
& \pi\left(z \mathcal{L}_{\sigma}^{\varsigma}(\varrho, \tau+1) f(z)\right)+(1-\pi)\left(z \mathcal{L}_{\sigma}^{\varsigma}(\varrho, \tau) f(z)\right) \prec \frac{1+\varpi z}{1+\eta z}+\frac{(\varpi-\eta) \pi z}{\tau(1+\eta z)^{2}}
\end{aligned}
$$

Then

$$
z \mathcal{L}_{\sigma}^{\varsigma}(\varrho, \tau) f(z) \prec \frac{1+\varpi z}{1+\eta z}, \quad z \in \mathfrak{D}
$$

and $\frac{1+\varpi z}{1+\eta z}$ is the best dominant of (3.26).

Proof Taking $\mathcal{J}(z)=\frac{1+\varpi z}{1+\eta z}$, we see that

$$
\Re\left(1+\frac{z \mathcal{J}^{\prime \prime}(z)}{\mathcal{J}^{\prime}(z)}\right)>\frac{1-|\eta|}{1+|\eta|}, \quad z \in \mathfrak{D}
$$

Hence, assumptions (3.18), (3.20), (3.22), and (3.24) imply that stipulations (3.1), (3.3), (3.5), and (3.7) are consistently in Theorem 3.1. Thus from Theorem 3.1 we acquire assertion (3.26).

Setting $\varpi=1$ and $\eta=-1$ in Theorem 3.2 yields the following.

Corollary 3.1 Let $\pi \in \mathcal{C}^{*}$ and $f \in \Sigma$. If any one of the following pairs of stipulations is achieved:

1.

$$
\begin{aligned}
& \Re\left(\frac{1}{\pi}\right)<0 \\
& \pi\left(z \mathcal{L}_{\sigma}^{\varsigma+1}(\varrho, \tau) f(z)\right)+(1-\pi)\left(z \mathcal{L}_{\sigma}^{\varsigma}(\varrho, \tau) f(z)\right) \prec \frac{1+z}{1-z}+\pi \vartheta \frac{2 z}{(1+z)^{2}}, \quad z \in \mathfrak{D},
\end{aligned}
$$

2.

$$
\begin{aligned}
& \Re\left(\frac{\varrho-1}{\pi}\right)>0, \\
& \pi\left(z \mathcal{L}_{\sigma}^{\varsigma}(\varrho-1, \tau) f(z)\right)+(1-\pi)\left(z \mathcal{L}_{\sigma}^{\varsigma}(\varrho, \tau) f(z)\right) \prec \frac{1+z}{1-z}+\frac{2 \pi z}{(\vartheta-1)(1+z)^{2}},
\end{aligned}
$$

3.

$$
\begin{aligned}
& \Re\left(\frac{1}{\pi}\right)>0, \\
& \pi\left(z \mathcal{L}_{\sigma+1}^{\varsigma}(\varrho, \tau) f(z)\right)+(1-\pi)\left(z \mathcal{L}_{\sigma}^{\varsigma}(\varrho, \tau) f(z)\right) \prec \frac{1+z}{1-z}+\frac{2 \pi z}{(\sigma+1)(1+z)^{2}}
\end{aligned}
$$


4.

$$
\begin{aligned}
& \Re\left(\frac{\tau}{\pi}\right)>0, \\
& \frac{1+z}{1-z}+\frac{2 \pi z}{\tau(1+z)^{2}}, \quad z \in \mathfrak{D} .
\end{aligned}
$$

Then

$$
z \mathcal{L}_{\sigma}^{\varsigma}(\varrho, \tau) f(z) \prec \frac{1+z}{1-z}
$$

and $\frac{1+z}{1-z}$ is the best dominant.

Theorem 3.3 Let $\mathcal{J}(z)$ be a nonzero univalent function in $\mathfrak{D}$ with $\mathcal{J}(0)=1, \gamma \in \mathcal{C}^{*}, \alpha, \beta \in$ $\mathcal{C}$, while $\alpha+\beta \neq 0$ and $f \in \Sigma$. Consider that $f$ and $\mathcal{J}$ attain the stipulations

$$
\frac{\alpha z \mathcal{L}_{\sigma+1}^{\varsigma}(\varrho, \tau) f(z)+\beta z \mathcal{L}_{\sigma}^{\varsigma}(\varrho, \tau) f(z)}{\alpha+\beta} \neq 0 \quad(z \in \mathfrak{D})
$$

and

$$
\Re\left\{1+\frac{z \mathcal{J}^{\prime \prime}(z)}{\mathcal{J}^{\prime}(z)}-\frac{z \mathcal{J}^{\prime}(z)}{\mathcal{J}(z)}\right\}>0 \quad(z \in \mathfrak{D})
$$

If

$$
\gamma\left[1+\frac{\alpha z\left(\mathcal{L}_{\sigma+1}^{\varsigma}(\varrho, \tau) f(z)\right)^{\prime}+\beta z\left(\mathcal{L}_{\sigma}^{\varsigma}(\varrho, \tau) f(z)\right)^{\prime}}{\alpha \mathcal{L}_{\sigma+1}^{\varsigma}(\varrho, \tau) f(z)+\beta \mathcal{L}_{\sigma}^{\varsigma}(\varrho, \tau) f(z)}\right] \prec \frac{z \mathcal{J}^{\prime}(z)}{\mathcal{J}(z)}
$$

then

$$
\frac{\alpha z \mathcal{L}_{\sigma+1}^{\varsigma}(\varrho, \tau) f(z)+\beta z \mathcal{L}_{\sigma}^{\varsigma}(\varrho, \tau) f(z)}{\alpha+\beta} \prec \mathcal{J}(z)
$$

and $\mathcal{J}$ is the best dominant of (3.29).

Proof From Lemma 2.2, we consider

$$
\begin{aligned}
& \varphi(\mathfrak{a})=0, \quad \rho(\mathfrak{a})=\frac{1}{\mathfrak{a}}, \\
& \mathfrak{P}(z)=z \mathcal{J}^{\prime}(z) \pi(\mathcal{J}(z))=\frac{z \mathcal{J}^{\prime}(z)}{\mathcal{J}(z)},
\end{aligned}
$$

and

$$
\mathcal{H}(z)=\mathfrak{P}(z) .
$$

By utilizing (3.27), $\mathfrak{P}(z)$ is univalent starlike in $\mathfrak{D}$. Further, we obtain

$$
\mathfrak{R}\left(\frac{z \mathcal{H}^{\prime}(z)}{\mathfrak{P}(z)}\right)>0 \quad(z \in \mathfrak{D}) .
$$


Next, let $\mathfrak{p}$ be a function formulated as follows:

$$
\mathfrak{p}(z)=\left[\frac{\alpha z \mathcal{L}_{\sigma+1}^{\varsigma}(\varrho, \tau) f(z)+\beta z \mathcal{L}_{\sigma}^{\varsigma}(\varrho, \tau) f(z)}{\alpha+\beta}\right]^{\gamma} \quad(z \in \mathfrak{D}) .
$$

Then $\mathfrak{p}$ is regular in $\mathfrak{D}$ with $\mathfrak{p}(0)=\mathcal{J}(0)=1$ and

$$
\frac{z \mathfrak{p}^{\prime}(z)}{\mathfrak{p}(z)}=\gamma\left[1+\frac{\alpha z\left(\mathcal{L}_{\sigma+1}^{\varsigma}(\varrho, \tau) f(z)\right)^{\prime}+\beta z\left(\mathcal{L}_{\sigma}^{\varsigma}(\varrho, \tau) f(z)\right)^{\prime}}{\alpha \mathcal{L}_{\sigma+1}^{\varsigma}(\varrho, \tau) f(z)+\beta \mathcal{L}_{\sigma}^{\zeta}(\varrho, \tau) f(z)}\right] .
$$

Using (3.31) in (3.28), we gain

$$
\frac{z \mathfrak{p}^{\prime}(z)}{\mathfrak{p}(z)} \prec \frac{z \mathcal{J}^{\prime}(z)}{\mathcal{J}(z)} .
$$

This means that

$$
\theta(\mathfrak{p}(z))+z \mathfrak{p}^{\prime}(z) \rho(\mathfrak{p}(z)) \prec \varphi(\mathcal{J}(z))+z \mathcal{J}^{\prime}(z) \pi(\mathcal{J}(z)) .
$$

So, from Lemma 2.2, we deduce

$$
\mathfrak{p}(z) \prec \mathcal{J}(z) \quad(z \in \mathfrak{D}),
$$

and $\mathcal{J}(z)$ is the best dominant. This is quite the assertion in (3.29).

Setting $\alpha=0, \beta=1$, and $\mathcal{J}(z)=\frac{1+\varpi z}{1+\eta z}$ in Theorem 3.3, it is clear to find out that hypothesis (3.27) holds whenever $-1 \leq \eta<\varpi \leq 1$, and therefore we acquire the next outcome.

Corollary 3.2 Let $-1 \leq \eta<\varpi \leq 1$ and $\gamma \in \mathcal{C}^{*}$. Let $f \in \Sigma$ and assume that

$$
z \mathcal{L}_{\sigma}^{\varsigma}(\varrho, \tau) f(z) \neq 0 \quad\left(z \in \mathfrak{D} ; \varsigma \in \mathcal{N}_{0} ; \sigma>-1\right) .
$$

If

$$
\gamma\left[1+\frac{z\left(\mathcal{L}_{\sigma}^{\varsigma}(\varrho, \tau) f(z)\right)^{\prime}}{\mathcal{L}_{\sigma}^{\varsigma}(\varrho, \tau) f(z)}\right] \prec \frac{(\varpi-\eta) z}{(1+\varpi z)(1+\eta z)}
$$

then

$$
\left[z \mathcal{L}_{\sigma}^{\varsigma}(\varrho, \tau) f(z)\right]^{\gamma} \prec \frac{1+\varpi z}{1+\eta z}
$$

and $\frac{1+\varpi z}{1+\eta z}$ is the best dominant of (3.32).

Theorem 3.4 If $f$ is univalent meromorphic starlike of order $\delta(0 \leq \delta<1)$ in $\mathfrak{D}^{*}$ and if $(1-\delta)=\frac{\varepsilon}{\gamma}, 0 \leq \varepsilon \leq 1$, then

$$
\left(z \mathcal{L}_{\sigma}^{\varsigma}(\varrho, \tau) f(z)\right)^{\gamma} \prec(1-z)^{2 \varepsilon}
$$

The function $(1-z)^{2 \varepsilon}$ is the best dominant of (3.33). Especially, $\left|z \mathcal{L}_{\sigma}^{\varsigma}(\varrho, \tau) f(z)\right|$ is bounded by $2^{2(1-\delta)}$ in $\mathfrak{D}$. 
Proof Since

$$
-\frac{z\left(\mathcal{L}_{\sigma}^{\varsigma}(\varrho, \tau) f(z)\right)^{\prime}}{\mathcal{L}_{\sigma}^{\varsigma}(\varrho, \tau) f(z)} \prec \frac{1+(1-2 \delta) z}{1-z} \quad(z \in \mathfrak{D}),
$$

we have

$$
\gamma\left[1+\frac{z\left(\mathcal{L}_{\sigma}^{\varsigma}(\varrho, \tau) f(z)\right)^{\prime}}{\mathcal{L}_{\sigma}^{\varsigma}(\varrho, \tau) f(z)}\right] \prec \frac{2 \varepsilon z}{1-z} .
$$

Therefore, taking $\alpha=\sigma=\varsigma=0, \beta=1, \varrho=\tau$, and $\mathcal{J}(z)=(1-z)^{2 \varepsilon}$ in Theorem 3.3, we gain (3.33). Thus, it is clear to achieve that $(1-z)^{2 \varepsilon}$ is univalent in $\mathfrak{D}$ if $0 \leq \varepsilon<1$.

Theorem 3.5 Let $\gamma \in \mathcal{C}^{*}$ and $\chi, \alpha, \beta \in \mathcal{C}$ with $\alpha+\beta \neq 0$. Let $\mathcal{J}$ be univalent in $\mathfrak{D}$ with $\mathcal{J}(0)=1$ and

$$
\Re\left(1+\frac{z \mathcal{J}^{\prime \prime}(z)}{\mathcal{J}^{\prime}(z)}\right)>\max \{0,-\Re(\chi)\} \quad(z \in \mathfrak{D}) .
$$

Assume that $f \in \Sigma$ attains

$$
\frac{\alpha z \mathcal{L}_{\sigma+1}^{\zeta}(\varrho, \tau) f(z)+\beta z \mathcal{L}_{\sigma}^{\zeta}(\varrho, \tau) f(z)}{\alpha+\beta} \neq 0 \quad(z \in \mathfrak{D}) .
$$

Set

$$
\begin{aligned}
E(z)= & {\left[\frac{\alpha z \mathcal{L}_{\sigma+1}^{\varsigma}(\varrho, \tau) f(z)+\beta z \mathcal{L}_{\sigma}^{\varsigma}(\varrho, \tau) f(z)}{\alpha+\beta}\right]^{\gamma} } \\
& \times\left[\chi+\gamma\left(\frac{\alpha z\left(\mathcal{L}_{\sigma+1}^{\varsigma}(\varrho, \tau) f(z)\right)^{\prime}+\beta z\left(\mathcal{L}_{\sigma}^{\varsigma}(\varrho, \tau) f(z)\right)^{\prime}}{\alpha \mathcal{L}_{\sigma+1}^{\varsigma}(\varrho, \tau) f(z)+\beta \mathcal{L}_{\sigma}^{\zeta}(\varrho, \tau) f(z)}+1\right)\right] .
\end{aligned}
$$

If

$$
\mathrm{E}(z) \prec \chi \mathcal{J}(z)+z \mathcal{J}^{\prime}(z)
$$

then

$$
\left[\frac{\alpha z \mathcal{L}_{\sigma+1}^{\varsigma}(\varrho, \tau) f(z)+\beta z \mathcal{L}_{\sigma}^{\varsigma}(\varrho, \tau) f(z)}{\alpha+\beta}\right]^{\gamma} \prec \mathcal{J}(z),
$$

and $\mathcal{J}$ is the best dominant of (3.36).

Proof The evidence for this theorem is similar to that of Theorem 3.3, and we draw major steps. Let $\mathfrak{p}(z)$ be a function given in (3.30). So, (3.31) leads to

$$
z \mathfrak{p}^{\prime}(z)=\gamma \mathfrak{p}(z)\left[1+\frac{\alpha z\left(\mathcal{L}_{\sigma+1}^{\varsigma}(\varrho, \tau) f(z)\right)^{\prime}+\beta z\left(\mathcal{L}_{\sigma}^{\varsigma}(\varrho, \tau) f(z)\right)^{\prime}}{\alpha \mathcal{L}_{\sigma+1}^{\varsigma}(\varrho, \tau) f(z)+\beta \mathcal{L}_{\sigma}^{\varsigma}(\varrho, \tau) f(z)}\right] .
$$

In this case, we take

$$
\varphi(\mathfrak{a})=\chi w, \quad \rho(\mathfrak{a})=1, \quad \mathfrak{a} \in \mathcal{C}
$$




$$
\mathfrak{P}(z)=z \mathcal{J}^{\prime}(z), \quad \rho(\mathcal{J}(z))=z \mathcal{J}^{\prime}(z) \quad(z \in \mathfrak{D}),
$$

and

$$
\mathfrak{p}(z)=\varphi(\mathfrak{P}(z))+\mathcal{J}(z)=\chi \mathcal{J}(z)+z \mathcal{J}^{\prime}(z), \quad(z \in \mathfrak{D})
$$

From (3.34), it yields $\mathfrak{P}(z)$ is starlike in $\mathfrak{D}$ and that

$$
\Re\left\{\frac{z \mathfrak{p}^{\prime}(z)}{\mathfrak{P}(z)}\right\}=\Re\left\{\chi+1+\frac{z \mathcal{J}^{\prime \prime}(z)}{\mathcal{J}^{\prime}(z)}\right\}>0
$$

Besides, by substituting the expressions for $\mathfrak{p}(z)$ and $z \mathfrak{p}^{\prime}(z)$ from (3.30) and from (3.37) consistently, we obtain

$$
\varphi(\mathfrak{p}(z))+z \mathfrak{p}^{\prime}(z) \rho(\mathfrak{p}(z))=\chi \mathfrak{p}(z)+z \mathfrak{p}^{\prime}(z)=\mathrm{E}(z)
$$

Assumption (3.35) is equivalent to

$$
\varphi(\mathfrak{p}(z))+z \mathfrak{p}^{\prime}(z) \rho(\mathfrak{p}(z)) \prec \varphi(\mathcal{J}(z))+z \mathcal{J}^{\prime}(z) \rho(\mathcal{J}(z)) .
$$

So Lemma 2.2 gains

$$
\mathfrak{p}(z) \prec \mathcal{J}(z)
$$

This last statement gets the assertion of (3.37).

Taking $\mathcal{J}(z)=\frac{1+\varpi z}{1+\eta z},(-1 \leq \eta<\varpi \leq 1), \alpha=0$, and $\beta=1$ in Theorem 3.5, we deduce the following.

Theorem 3.6 Let $\gamma \in \mathcal{C}^{*},-1 \leq \eta<\varpi \leq 1$, and

$$
\mathfrak{a}=\frac{|\eta|-1}{|\eta|+1}
$$

Iff $\in \Sigma$ attains

$$
z \mathcal{L}_{\sigma}^{\mathcal{S}}(\varrho, \tau) f(z) \neq 0 \quad(z \in \mathfrak{D})
$$

and

$$
\left[z \mathcal{L}_{\sigma}^{\varsigma}(\varrho, \tau) f(z)\right]^{\gamma}\left[\chi+\gamma\left(1+\frac{z\left(\mathcal{L}_{\sigma}^{\varsigma}(\varrho, \tau) f(z)\right)^{\prime}}{\mathcal{L}_{\sigma}^{\varsigma}(\varrho, \tau) f(z)}\right)\right] \prec \chi \frac{1+\varpi z}{1+\eta z}+\frac{(\varpi-\eta) z}{(1+\eta z)^{2}}
$$

then

$$
\left[z \mathcal{L}_{\sigma}^{\varsigma}(\varrho, \tau) f(z)\right]^{\gamma} \prec \frac{1+\varpi z}{1+\eta z}
$$

and $\frac{1+\varpi z}{1+\eta z}$ is the best dominant of (3.40). 
Proof In this case

$$
\Re\left(1+\frac{z \mathcal{J}^{\prime \prime}(z)}{\mathcal{J}^{\prime}(z)}\right)=\Re\left\{\frac{1-\eta z}{1+\eta z}\right\}>\frac{1-|\eta|}{1+|\eta|} \quad(z \in \mathfrak{D}) .
$$

Therefore, stipulation (3.34) gives (3.38). By utilizing Theorem 3.5, we conclude that assertion (3.40) holds.

Again setting $\alpha=1, \beta=\sigma=\varsigma=\chi=0, a=\tau$, and $\mathcal{J}(z)=\frac{1+z}{1-z}$ in Theorem 3.6, we get the following.

Corollary 3.3 Let $f \in \Sigma$ be such that $z f(z) \neq 0$ for $z \in \mathfrak{D}$, and let $\gamma \in \mathcal{C}^{*}$. If

$$
(z f(z))^{\gamma}\left[\gamma\left(1+\frac{z f^{\prime}(z)}{f(z)}\right)\right] \prec \frac{2 z}{(1-z)^{2}}
$$

then

$$
(z f(z))^{\gamma} \prec \frac{1+z}{1-z}
$$

and $\frac{1+z}{1-z}$ is the best dominant of $(3.41)$.

\section{Conclusion}

Special functions such as Hurwitz-Lerch zeta (HL-Z) functions have been continuously developed. Indeed, the theme of developments formula for HL-Z functions and correlated functions has a long history, which can be traced back to Goldbach and Euler [14]. This research looked at a convolution complex operator in complex punctured unit disk formulated for meromorphic regular functions. Based on the said operator, a new subclass of meromorphic functions is studied. Differential inequalities are investigated utilizing the theory of first-order subordination. Inclusion and subordination theorems are obtained using the techniques of differential subordination and identities utilizing the HurwitzLerch zeta function. Numerous intriguing special cases of the fundamental theorems are highlighted in the form of corollaries. The proposed operators can be employed to generalize other types of convolution, differential, and integral operators such as fractional operators or to establish several classes of normalized regular functions.

\section{Acknowledgements}

Not applicable.

Funding

This research was funded by the University of Sharjah, project ID: 2002144093.

Availability of data and materials

Not applicable.

\section{Declarations}

Competing interests

The authors declare that they have no competing interests. 


\section{Author details}

${ }^{1}$ Department of Mathematics, College of Sciences, University of Sharjah, Sharjah, United Arab Emirates. ${ }^{2}$ Department of Mathematics, College of Sciences, University of Baghdad, Baghdad, Iraq. ${ }^{3}$ Department of Mathematics, Faculty of Science, Hadhramout University, Hadhramout, 50512, Yemen.

\section{Publisher's Note}

Springer Nature remains neutral with regard to jurisdictional claims in published maps and institutional affiliations.

Received: 28 September 2021 Accepted: 2 February 2022 Published online: 19 February 2022

\section{References}

1. Goodman, A.W.: Univalent Functions, I. Mariner, Tampa (1983)

2. Miller, S.S., Mocanu, P.T.: Differential Subordinations: Theory and Applications. Dekker, New York (1999)

3. Miller, S.S., Mocanu, P.T.: Subordinants of differential superordinations. Complex Var. Theory Appl. 48(10), 815-826 (2003)

4. Alexander, J.W.: Functions which map the interior of the unit circle upon simple regions. Ann. Math. 17(1), 12-22 (1915)

5. Libera, R.J.: Some classes of regular univalent functions. Proc. Am. Math. Soc. 16, 755-758 (1965)

6. Ruscheweyh, S.: New criteria for univalent functions. Proc. Am. Math. Soc. 49, 109-115 (1975)

7. Pfaltzgraff, J.: Univalence of the integral of $f^{\prime}(z)^{\lambda}$. Bull. Lond. Math. Soc. 7(3), 254-256 (1975)

8. Pascu, N.N., Pescar, V.: On integral operators of Kim-Merkes and Pfaltzgraff. Mathematica (Cluj) 32(55), 185-192 (1990)

9. Sălăgean, G.S.: Subclasses of univalent functions. Lect. Notes Math. 1013, 362-372 (1983)

10. Carlson, B.C., Shaffer, D.B.: Starlike and prestarlike hypergeometric functions. SIAM J. Math. Anal. 15, 737-745 (1984)

11. Srivastava, H.M., Owa, S. (eds.): Univalent Functions, Fractional Calculus, and Their Applications Halsted, New York (1989)

12. Srivastava, H.M., Attiya, A.A.: An integral operator associated with the Hurwitz-Lerch zeta function and differential subordination. Integral Transforms Spec. Funct. 18(3), 207-216 (2007)

13. Srivastava, H.M., Saxena, R.K., Pogány, T.K., Saxena, R.: Integral and computational representations of the extended Hurwitz-Lerch zeta function. Integral Transforms Spec. Funct. 22, 487-506 (2011)

14. Srivastava, H.M.: The zeta and related functions: recent developments. J. Adv. Engrg. Comput. 3, 329-354 (2019)

15. Srivastava, H.M.: Riemann, Hurwitz and Hurwitz-Lerch zeta functions and associated series and integrals. In: Pardalos, P., Rassias, T. (eds.) Essays in Mathematics and Its Applications. Springer, Berlin (2012)

16. Srivastava, H.M.: Some general families of the Hurwitz-Lerch zeta functions and their applications: recent developments and directions for further researches. Proc. Inst. Math. Mech. Natl. Acad. Sci. Azerb. 45, 234-269 (2019)

17. Srivastava, H.M.: Operators of basic (or q-) calculus and fractional q-calculus and their applications in geometric function theory of complex analysis. Iran. J. Sci. Technol. Trans. A, Sci. 44, 327-344 (2020)

18. Srivastava, H.M., Wanas, A.K., Srivastava, R.: Applications of the q-Srivastava-Attiya operator involving a certain family of bi-univalent functions associated with the Horadam polynomials. Symmetry 13(1230), 1-14 (2021)

19. Srivastava, H.M., Raza, N., AbuJarad, E.S.A., Srivastava, G., AbuJarad, M.H.: Fekete-Szegö inequality for classes of $(p, q)$-starlike and $(p, q)$-convex functions. Rev. R. Acad. Cienc. Exactas Fís. Nat., Ser. A Mat. 113, 3563-3584 (2019)

20. Dziok, J., Srivastava, H.M.: Certain subclasses of analytic functions associated with the generalized hypergeometric function. Integral Transforms Spec. Funct. 14(1), 7-18 (2003)

21. Ghanim, F.: A study of a certain subclass of Hurwitz-Lerch zeta function related to a linear operator. Abstr. Appl. Anal. 2013, Article ID 763756 (2013). https://doi.org/10.1155/2013/763756

22. Ghanim, F.: Certain properties of classes of meromorphic functions defined by a linear operator and associated with Hurwitz-Lerch zeta function. Adv. Stud. Contemp. Math. 27, 175-180 (2017)

23. Challab, K.A., Darus, M., Ghanim, F.: On subclass of meromorphically univalent functions defined by a linear operator associated with $\boldsymbol{\lambda}$-generalized Hurwitz-Lerch zeta function and q-hypergeometric function. Ital. J. Pure Appl. Math. 39, 410-423 (2018)

24. Ghanim, F., Al-Shaqsi, K., Darus, M., Al-Janaby, H.F.: Subordination properties of meromorphic Kummer function correlated with Hurwitz-Lerch zeta-function. Mathematics 9, 192 (2021)

25. Ghanim, F., Al-Janaby, H.F.: A certain subclass of univalent meromorphic functions defined by a linear operator associated with the Hurwitz-Lerch zeta function, Rad HAZU. Rad Hrvat. Akad. Znan. Umjet. Mat. Znan. 23, 71-83 (2019)

26. Al-Janay, H.F., Ghanim, F., Darus, M.: Some geometric properties of integral operators proposed by Hurwitz-Lerch zeta function. J. Phys. Conf. Ser. 1212, 1-6 (2019)

27. Al-Janaby, H.F., Ghanim, F., Darus, M.: On the third-order complex differential inequalities of $\xi$-generalized-Hurwitz-Lerch zeta functions. Mathematics 8, Article ID 845 (2020) https://doi.org/10.3390/math8050845

28. Srivastava, H.M., Jolly, N., Bansal, M.K., Jain, R.: A new integral transform associated with the $\lambda$-extended Hurwitz-Lerch zeta function. Rev. R. Acad. Cienc. Exactas Fís. Nat., Ser. A Mat. 113, 1679-1692 (2019)

29. Srivastava, H.M., Ghanim, F., El-Ashwah, R.M.: Inclusion properties of certain subclass of univalent meromorphic functions defined by a linear operator associated with the $\lambda$-generalized Hurwitz-Lerch zeta function. Bul. Acad. Ştiinţe Repub. Mold. Mat. 3(85), 34-50 (2017)

30. Mishra, A.K., Panigrahi, T., Mishra, R.K.: Subordination and inclusion theorems for subclasses of meromorphic functions with applications to electromagnetic cloaking. Math. Comput. Model. 57, 945-962 (2013)

31. Srivastava, H.M., Gaboury, S., Ghanim, F.: Partial sums of certain classes of meromorphic functions related to the Hurwitz-Lerch zeta function. Moroccan J. Pure Appl. Anal. 1, 38-50 (2015)

32. Srivastava, H.M., Jankov, D., Pogány, T.K., Saxena, R.K.: Two-sided inequalities for the extended Hurwitz-Lerch zeta function. Comput. Math. Appl. 62(1), 516-522 (2011)

33. Srivastava, H.M., Choi, J.: Series Associated with the Zeta and Related Functions. Kluwer Academic, Dordrecht (2001) 
34. Srivastava, H.M.: A survey of some recent developments on higher transcendental functions of analytic number theory and applied mathematics. Symmetry 13, Article ID 2294 (2021)

35. Srivastava, H.M., Gaboury, S., Ghanim, F.: Certain subclasses of meromorphically univalent functions defined by a linear operator associated with the $\lambda$-generalized Hurwitz-Lerch zeta function. Integral Transforms Spec. Funct. 26(4), 258-272 (2015)

36. Srivastava, H.M., Gaboury, S., Ghanim, F.: Some further properties of a linear operator associated with the $\lambda$-generalized Hurwitz-Lerch zeta function related to the class of meromorphically univalent functions. Appl. Math. Comput. 259, 1019-1029 (2015)

37. Wilken, D.R., Feng, J.: A remark on convex and starlike functions. J. Lond. Math. Soc. s2-21(2), 287-290 (1980)

38. Whittaker, E.T., Watson, G.N.: A Course on Modern Analysis: An Introduction to the General Theory of Infinite Processes and of Analytic Functions, with an Account of the Principal Transcendental Functions, 4th edn. Cambridge University Press, Cambridge (1962)

\section{Submit your manuscript to a SpringerOpen ${ }^{\circ}$ journal and benefit from:}

- Convenient online submission

- Rigorous peer review

- Open access: articles freely available online

- High visibility within the field

- Retaining the copyright to your article

Submit your next manuscript at $\gg$ springeropen.com 